\title{
Curious Case of Rodent Poison: A Case Report
}

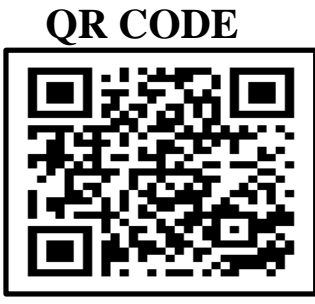

\section{SAM PONRAJ DAVIDSON. S*1, SOMASUNDARAM ELANGOVAN², SUMAN JHANSI LAKSHMI ${ }^{3}$, B. SENTHIL KUMAR ${ }^{3}$}

Osteonecrosis was first described as a consequence of ionizing radiation used in the treatment of malignant tumors but later a variety of causes for osteonecrosis were found which include, Medication induced, radiation induced, chemical induced and infection induced. Terms such as: bisphosphonate osteonecrosis (BON), Bisphosphonate Associated Osteonecrosis of the Jaws (BONJ), Bisphosphonate Related Osteonecrosis of the Jaws (BRONJ), Antiresorptive Osteonecrosis of the Jaw (ARONJ) and Medication Related Osteonecrosis of the Jaw (MRONJ) have all had proponents and all continue to be utilized. However, Migliorati first proposed a designation of the disease as osteochemonecrosis. This case report is about a chemical induced osteonecrosis patient who placed rat poison inside the mouth.

KEYWORDS: Osteonecrosis, Rat Poison, BRONJ, MRONJ

\section{INTRODUCTION}

Osteonecrosis of the jaw (ONJ) was previously defined as exposed, necrotic bone in the maxillofacial region for at least 8 weeks in patients receiving an antiresorptive medication for primary or metastatic bone cancer, osteoporosis, or Paget's disease, with no previous radiation therapy to the jaws. ${ }^{1,2}$ Recently, the AAOMS revised the definition to include exposed bone, or bone that can be probed through an intraoral or extraoral fistula in patients on antiresorptive or antiangiogenic medications. ${ }^{3}$ Osteonecrosis was first described as an outcome of ionizing radiation which was used in the management of malignant tumors. ${ }^{4}$ Later, osteonecrosis came upon as an outcome of the prolonged use of some varieties from the bisphosphonates (BPs) and lately as a result of the use of medications which act on bone remodelling and anti-angiogenesis..$^{5-7}$ Here we report a case of a 36-yearold female patient corresponding to the clinical and histopathological features of Osteonecrosis in the palatal region.

\section{CASE REPORT}

A 36-year-old female patient came to our department with a chief complaint of exposure of bone in upper front tooth region of the jaw for past 1 year. History revealed that patient had placed the rat poison mixed in papaya fruit in the mouth for sometime and had spat it out apparently because of change of mind. She was rushed to the hospital for emergency management and gastric lavage was done. Two days after hospitalization she noticed the upper front teeth have become mobile and they fell off by themselves subsequently and she also noticed that one half of roof of the mouth had become black in colour. After discharge she consulted a private dental practitioner who removed the blackened tissue completely. After few days patient became aware of exposure of the bone as she could feel the sharp edges with her tongue but there was no pain or discomfort. She consulted few dental practitioners during the past 1 year. When surgical management was advised she was not willing for treatment. One of the dental practitioners referred her to our institution. She had no apparent systemic illness, no drug history and no deleterious habits.

On examination there was an exposure of bone on the palatal aspect from 21 to 25 . The junction between the exposed bone and the adjacent palatal mucosa was well demarcated (figure 1). Exposure of bone on the labial aspect of 22 to 25, destruction of labial cortex in 22,23 with pus discharge was also seen (figure 2). The exposed bone appeared avascular and necrotic. On palpation, the patient had no tenderness anywhere on the exposed bone but the patient had sensation and could feel the touch. With all the clinical findings and the history, a provisional diagnosis of osteonecrosis due to contact with harmful chemical was given and the differential diagnosis of mucormycosis and osteomyelitis were considered.

Regarding the investigation an OPG was taken and the visibility of the outline of sockets in relation to $22 \& 23$ even after 1 year suggestive of unhealed non-vital bone (figure 3). A PNS view was taken and there was no 


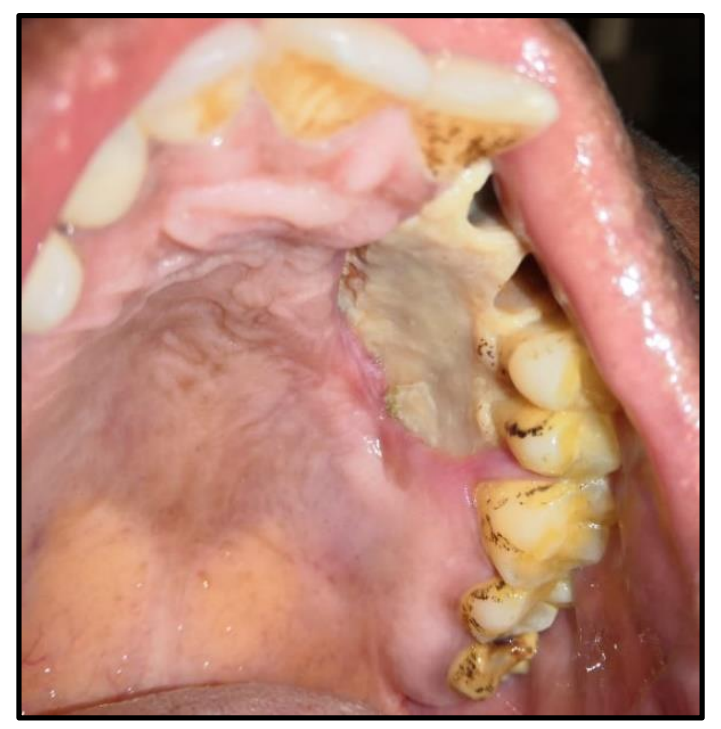

Figure 1. Exposure of bone seen on the palatal aspect with well-defined borders between exposed bone and palatal mucosa

evidence of involvement into the nasal cavity, maxillary sinus or other adjacent structures. Hematological parameters were within normal limits. Cytological smears were taken from the exposed bone and a few fragments of bone were removed and were sent to Department of Oral Pathology for microscopic examination and the results showed no evidence of fungal hyphae. Then a bone biopsy was done and sent to Department of Oral Pathology and the results showed it as a necrotic bone because it showed bone trabeculae with empty lacunae (figure 4).

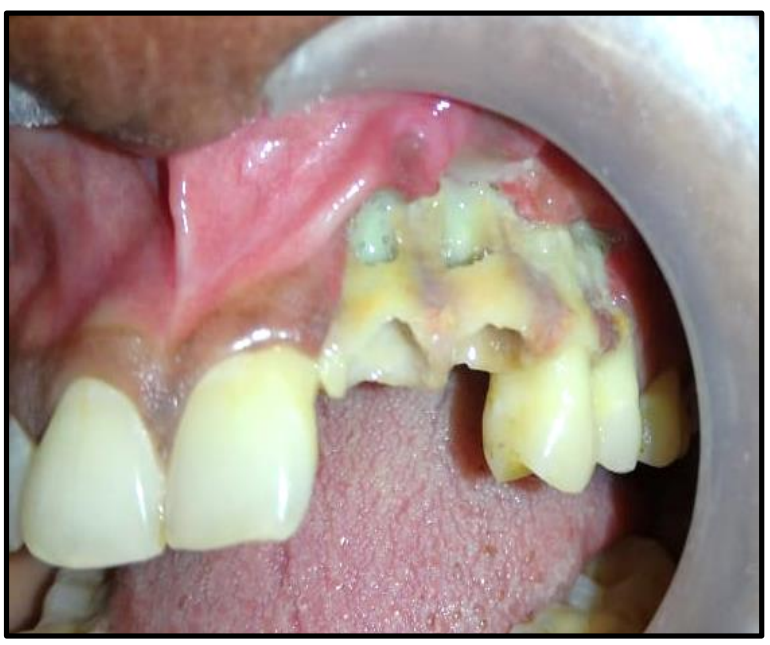

Figure 2. Destructed labial cortex with pus discharge

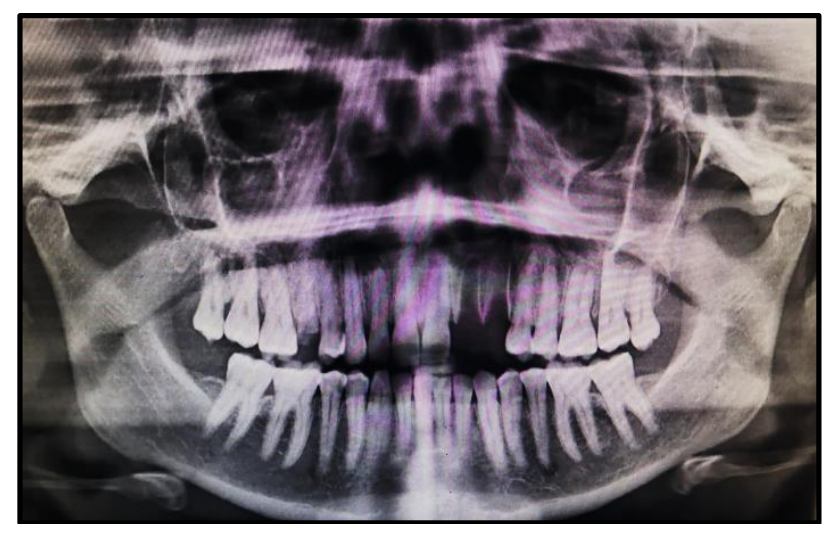

Figure 3. OPG showing outline of sockets in 22 and 23 even though it was extracted 1 year ago suggesting unhealed non vital bone

With all the clinical, radiographic and histopathologic findings, a final diagnosis of chemical induced osteonecrosis of the left maxilla was given and the patient was referred to the department of oral and maxillofacial surgery for further management. Surgical resection was done on the left side of the palate and the healing was good (figure 5) and the patient was on regular follow up.

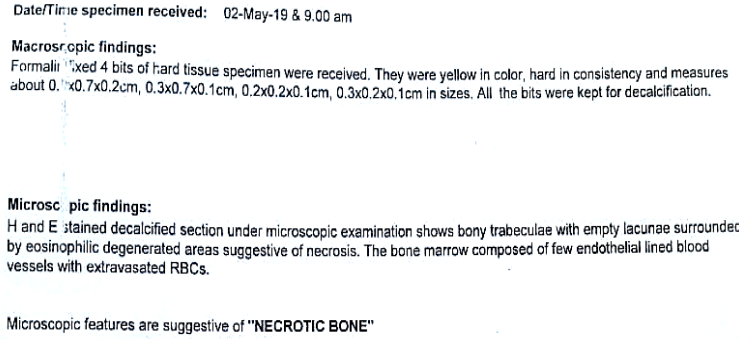

Figure 4. Bone biopsy report

\section{DISCUSSION}

Osteonecrosis of the jaw (ONJ) is described as a bone infarction due to ischemia. It is a clinically devastating condition that can affect the patient's quality of life. The main predisposing factors for ONJ are surgical intervention and oral mucosa breakdown in patients encountering to radiotherapy and chemotherapy agents. ${ }^{8}$ Medication-related osteonecrosis of the jaws (MRONJ) can also be defined clinically by necrotic bone exposure nevertheless, clinical manifestations without the exposure of bone, such as hypoesthesia/numbness of lower lip (Vincent's symptom), deep periodontal pocket, trismus, loose tooth and non-odontogenic pain.5,6 


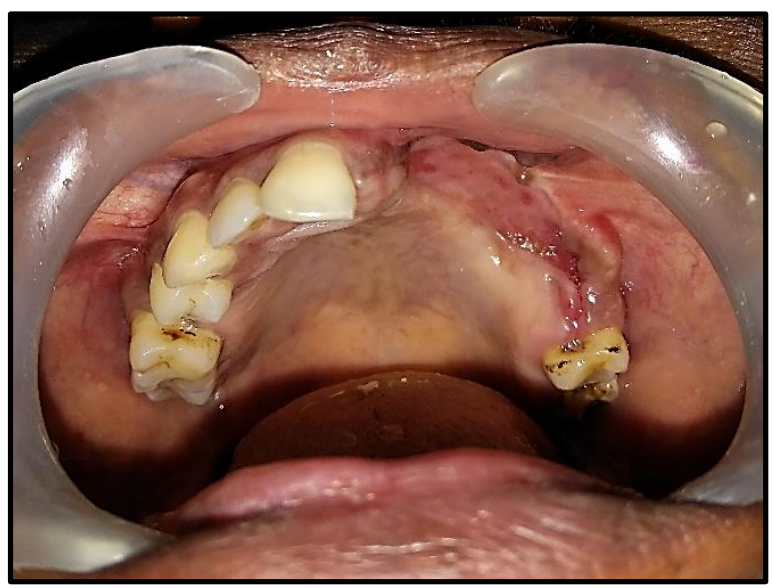

Figure 5. Post operative clinical picture showing good healing

Migliorati first proposed the term osteochemonecrosis. This term continues to be prestige because of the increasing numbers of drugs coorrelated with the disease, as well as its similarity to the designation of osteoradionecrosis. ${ }^{9}$

American Association of Oral and Maxillofacial Surgeons (AAOMS) proposed a staging for system is that the patients should be on an anti-resorption drug, should have no previous history of Head \& Neck radiation therapy or metastatic diseases in the jaws and should have signs and/or symptoms of duration of at least 8 weeks. The reason for this 8 weeks duration is that it takes longer time period for the bone to demonstrate evidence of associated osteolytic or sclerotic changes in the radiograph.

Treatment strategies continue to stress prevention and others include antiseptic rinses, systemic antibiotics, and cleaning or removal of dead bone from the affected area. Generally, therapy focuses on controlling pain and preventing infection so that the body can heal properly. ${ }^{10}$

\section{CONCLUSION}

$\mathrm{ONJ}$ is a multifactorial disease in patients with primary or metastatic bone malignancy or osteoporosis undergoing systemic antiresorptive therapy, where the pathophysiology has not yet been fully determined.

Other than Drugs and radiation some chemicals can also cause osteonecrosis of the jaw. In the present case osteonecrosis occurred because of the contact of palatal mucosa with the rat poison and the mucosa was sloughed off with the bone exposed. In this case, surgery was the only option. More awareness is needed for the public besides doctors about the ill effects of chemicals and the resulting osteonecrosis and the early diagnosis with faster and accurate treatment is mandatory to save the vital structures of the oral cavity.

\section{REFERENCES}

1. Khan AA, Morrison A, Hanley DA, Felsenberg D, McCauley LK, O’Ryan F, et al. International Task Force on Osteonecrosis of the J. Diagnosis and management of osteonecrosis of the jaw: a systematic review and international consensus. J Bone Miner Res. 2015;30(1):323. https://doi.org/10.1002/jbmr.2405

2. Ruggiero SL, Dodson TB, Assael LA, Landesberg R, Marx RE, Mehrotra B. American Association of Oral and Maxillofacial Surgeons position paper on bisphosphonate-related osteonecrosis of the jaws-2009 update. J Oral Maxillofac Surg. 2009;67:2.

3. Ruggiero SL, Dodson TB, Fantasia J, Goodday R, Aghaloo T, Mehrotra B, O'Ryan F. American Association of Oral and Maxillofacial Surgeons Position Paper on Medication-Related Osteonecrosis of the Jaw2014 Update. J Oral Maxillofac Surg. 2014;72:1938.

4. Marx RE. Osteoradionecrosis: a new concept of its pathophysiology. J Oral Maxillofac Surg. 1983;41:283-8. 5. Ruggiero SL, Dodson TB, Fantasia J, Goodday R, Aghaloo T, Mehrotra B, et al. American Association of Oral and Maxillofacial Surgeons position paper on medication-related osteonecrosis of the jaw - 2014 update. J Oral Maxillofac Surg. 2014;72:1938-56. https://doi.org/10.1016/j.joms.2014.04.031

6. Yoneda T, Hagino H, Sugimoto T, Ohta H, Takahashi $\mathrm{S}$, Soen S, et al. Antiresorptive agent-related osteonecrosis of the jaws: Position Paper 2017 of the Japanese Allied Committee on Osteonecrosis of the Jaw. J Bone Miner Metab. 2017;35:6-19. https://doi.org/10.1007/soo774-016-0810-7

7. Fusco V, Santini D, Armento G, Tonini G, Campisi G. Osteonecrosis of jaw beyond antiresorptive (bonetargeted) agents: New horizons in oncology. Expert Opin Drug Saf. 2016;15:925-35

8. Hoff AO, Toth BB, Altundag $\mathrm{K}$, Johnson MM, Warneke CL, Hu M, et al. Frequency and risk factors associated with osteonecrosis of the jaw in cancer patients treated with intravenous bisphosphonates. J Bone Miner Res. 2008;23:826-36.

9. Migliorati CA. Bisphosphanates and oral cavity avascular bone necrosis. J Clin Oncol. 2003;21(22):42534254. https://doi.org/10.1200/JCO.2003.99.132. 
10. Morrish RB Jr., Silverman CE, Jr., Meyer J, Fu KK, Greenspan D. Osteonecrosis in patients irradiated for head and neck carcinoma. Cancer. 1981;47:1980.

Cite this article as:

Davidson SSSP, Elangovan S, Jaishankar S, Kumar BS. Curious Case of Rodent Poison: A Case Report. Int Healthc Res J. 2021;5(9):CR1-CR4. https://doi.org/10.26440/IHRJ/0509.12484

\section{AUTHOR AFFILIATIONS: (*Corresponding Author)}

1. MDS, Senior Lecturer

2. Professor \& H.O.D

3. Professor

Department of Oral Medicine and Radiology, The Tamil Nadu Dr. M.G.R Medical University, Chennai 